ARTICLE

DOI: $10.1038 / \mathrm{s} 41467-017-02331-4$

\title{
Impact of grain boundaries on efficiency and stability of organic-inorganic trihalide perovskites
}

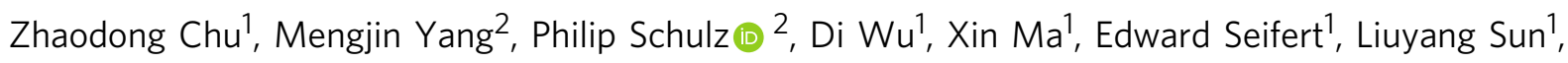
Xiaoqin $\mathrm{Li}^{1}$, Kai Zhu ${ }^{2}{ }^{2}$ \& Keji Lai ${ }^{1}$

Organic-inorganic perovskite solar cells have attracted tremendous attention because of their remarkably high power conversion efficiencies. To further improve device performance, it is imperative to obtain fundamental understandings on the photo-response and long-term stability down to the microscopic level. Here, we report the quantitative nanoscale photoconductivity imaging on two methylammonium lead triiodide thin films with different efficiencies by light-stimulated microwave impedance microscopy. The microwave signals are largely uniform across grains and grain boundaries, suggesting that microstructures do not lead to strong spatial variations of the intrinsic photo-response. In contrast, the measured photoconductivity and lifetime are strongly affected by bulk properties such as the sample crystallinity. As visualized by the spatial evolution of local photoconductivity, the degradation process begins with the disintegration of grains rather than nucleation and propagation from visible boundaries between grains. Our findings provide insights to improve the electrooptical properties of perovskite thin films towards large-scale commercialization.

\footnotetext{
${ }^{1}$ Department of Physics, University of Texas at Austin, Austin, TX 78712, USA. ${ }^{2}$ National Renewable Energy Laboratory, Golden, CO 80401 , USA. Zhaodong Chu and Mengjin Yang contributed equally to this work. Correspondence and requests for materials should be addressed to

X.L. (email: elaineli@physics.utexas.edu) or to K.Z. (email: kai.zhu@nrel.gov) or to K.L. (email: kejilai@physics.utexas.edu)
} 
T he worldwide surge of research interest in organic-inorganic trihalide perovskites, e.g., methylammonium lead triiodide $\left(\mathrm{CH}_{3} \mathrm{NH}_{3} \mathrm{PbI}_{3}\right.$ or $\left.\mathrm{MAPbI}_{3}\right)$, has led to a phenomenal increase of the power conversion efficiency (PCE) of perovskite solar cells (PSCs) from 3.8 to $22 \%$ in the past few years ${ }^{1-6}$. These hybrid organic-inorganic thin films are polycrystalline in nature and compatible with low-cost solution or vapor-based processes ${ }^{7-9}$. Yet their performance rivals many single-crystalline semiconductor solar cells ${ }^{10}$ owing to a number of intriguing optical and electrical properties that are ideal for energy harvesting and charge transport, such as high absorption coefficient across the visible spectrum ${ }^{11}$, high carrier mobility $^{11,12}$, and long carrier recombination lifetime ${ }^{13,14}$. The unprecedented progress of PSC efficiency was often attributed to the unique defect structures in the bulk and the benign grain boundaries during the early stage of PSC development ${ }^{15,16}$.

As the PSC efficiency continues to increase, recent efforts on increasing grain sizes and/or passivating grain boundaries (GBs) have casted doubts on the general belief in the unique defect tolerance in perovskites. Several groups including us have found that, when the grain size is increased from a few hundred nanometers to the micrometer level, the device performance is often significantly improved together with elongated charge-carrier lifetimes ${ }^{17-21}$. At first sight, these studies imply that GBs in polycrystalline perovskite thin films may not be as benign as early studies had suggested. A recent theoretical study pointed out that GBs may even be the major recombination sites in the standard iodide based perovskites ${ }^{22}$, which seems to be consistent with the recent experimental efforts described above. It is worth noting that regardless the chemical/physical natures of the defects, there are three primary spatial locations of defects related to perovskite thin films, i.e., film surface, bulk of the grain, and boundary between neighboring grains. Thus, in addition to possible changes of GB properties, the various new growth controls for increasing grain sizes could also affect the surface and bulk properties of perovskite grains, e.g., enhanced crystallinity, reduced defect density at the surface and in the bulk, and reduced structural defects associated with pinhole formation. Thus, it is important to scrutinize and isolate the impact of these different microscopic factors on electro-optical properties of polycrystalline perovskite thin films. Moreover, as material stability continues to be the key challenge faced by the PSC community ${ }^{8}$, an immediate question is whether the GB and/or the surface of perovskite films are the weakest points where the degradation would start first. To this end, understanding the degradation mechanism at the microscopic level is also imperative for fabricating robust and reliable devices that meet the stringent requirements of commercialization $^{23-29}$. In contrast to conventional macroscopic device characterizations, it is expected that spatially resolved studies on the chemical, electrical, and optical properties of the PSC thin films will provide crucial information for advancing the basic science and developing commercial products based on these fascinating materials.

While a number of scanning probe techniques ${ }^{30-38}$ have been used to interrogate properties of the PSC, local measurements of the intrinsic photoconductivity, rather than the extrinsic photocurrent across the Schottky-like tip-sample junction, have not been reported to investigate the role of various microstructures on the films. In addition, due to the poor air stability of the organic-inorganic trihalide compound, experiments on samples with a surface encapsulation layer are desirable to obtain reliable results. A non-contact method capable of mapping out the nanoscale photoconductivity on encapsulated films is thus particularly important for the PSC research. In this work, we report the first quantitative microwave impedance imaging with light stimulation on two $\mathrm{MAPbI}_{3}$ thin films with different PCEs capped by a polymethyl methacrylate (PMMA) protection layer. The photo-response is spatially uniform across grains and grains boundaries, whereas the difference of carrier mobility and lifetime between these two films is attributed to the difference of sample crystallinity. Both the surface topography and local photoconductivity have been monitored over an extended period of time, which sheds new light on the intricate degradation process of the PSC devices.

\section{Results}

Microwave photoconductivity imaging on $\mathrm{MAPbI}_{3}$. Our experiments are performed on a unique microwave impedance microscopy (MIM) $)^{39,40}$ setup with a focused laser beam illuminating from below the sample stage, as illustrated in Fig. 1a. The bottom illumination scheme avoids light being shallowed by the body of the cantilever probe ${ }^{41}$ and ensures an accurate calibration of the areal power intensity on the sample. Taking advantage of the near-field interaction, we are able to obtain a spatial a

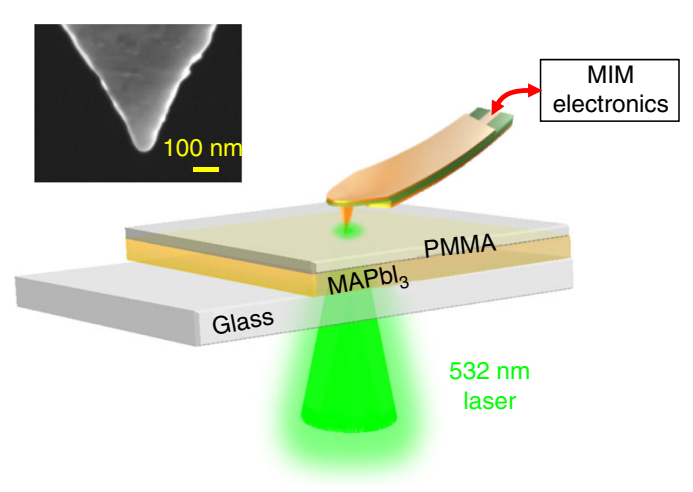

b

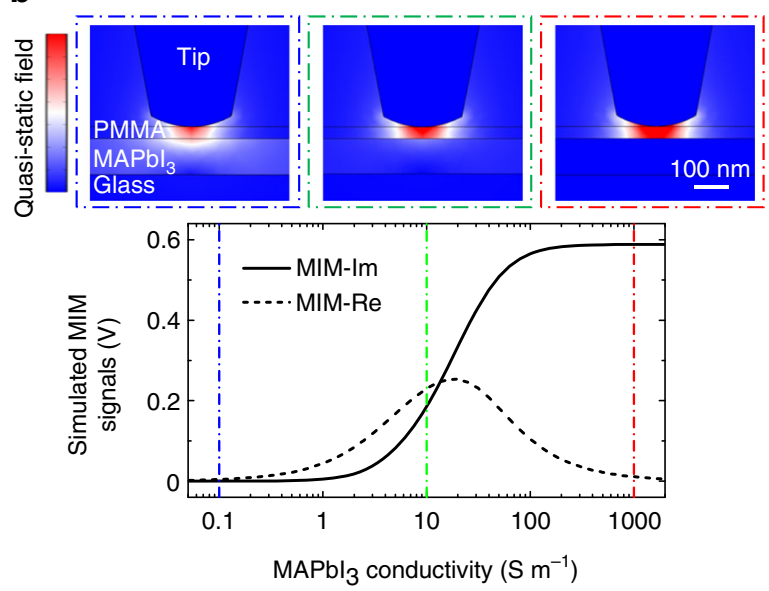

Fig. 1 Experiment setup and finite-element analysis. a Schematic diagram of the sample and the MIM setup with bottom illumination through the transparent glass substrate. Scanning is accomplished by moving the sample stage while fixing the laser beam and the probe tip, which are aligned before the experiment. The inset shows the scanning electron microscopy (SEM) image of a typical MIM tip. $\mathbf{b}$ Simulated MIM signals as a function of the MAPbl 3 conductivity $\sigma$. The quasi-static displacement-field distributions when $\sigma$ is equal to $0.1,10$, and $1000 \mathrm{~S} \mathrm{~m}^{-1}$ are displayed inside the blue, green, and red dash-dotted boxes, respectively 
resolution with deeply subwavelength lateral dimensions, i.e., on the order of the tip diameter (around $100 \mathrm{~nm}$ or $1 / 30,000 \lambda$, where $\lambda$ is the wavelength of the $1 \mathrm{GHz}$ microwave ${ }^{40}$. Here the $1 \mathrm{GHz}$ signal is delivered to the shielded cantilever tip ${ }^{42}$ and the reflected microwave is amplified and demodulated to form MIM-Im and MIM-Re signals, which are proportional to the imaginary and real parts of the tip-sample admittance ${ }^{39}$. Unlike the conductive $\mathrm{AFM}^{32-35}$, a direct contact between the MIM tip and the perovskite thin films is not required due to the efficient capacitive coupling at $\mathrm{GHz}$ frequencies, overcoming the challenges of interrogating nanoscale electrical properties on samples with an insulating capping layer. The contrast mechanism of the MIM is similar to that of the time-resolved microwave conductivity (TRMC) experiment ${ }^{11,12,43-46}$, a non-contact technique widely exploited to study the dynamics of photo-generated carriers, except that the time resolution in TRMC under pulsed excitation is traded for spatial resolution in MIM under continuous illumination.

The $100-\mathrm{nm}$ thick $\mathrm{MAPbI}_{3}$ thin films are prepared by an excess organic salt based solvent-solvent extraction method ${ }^{21}$. The precursor solution $(30 \mathrm{wt} \%)$ is spin-coated on glass substrates at $6000 \mathrm{rpm}$ for $25 \mathrm{~s}$ to form a wet precursor film, which is then transferred into a stirred ether bath for $1 \mathrm{~min}$, followed by thermal annealing at $150{ }^{\circ} \mathrm{C}$ for $15 \mathrm{~min}$ with a petri dish covered. Solar cell devices made from the same material but with thicker film $(350 \mathrm{~nm})$ have demonstrated a PCE of $18 \%$ (reverse scan) under the standard Air Mass (AM) 1.5 illumination (Supplementary Fig. 1). As a comparison, 100-nm thick $\mathrm{MAPbI}_{3}$ films with smaller grain size was deposited using the same process except with stoichiometry precursor and annealing at $100^{\circ} \mathrm{C}$ for $10 \mathrm{~min}$. Devices with small grain size and thicker $(350 \mathrm{~nm})$ perovskite film possess a PCE of $15 \%$ (reverse scan) (Supplementary Fig. 1). To further verify the cell performance, we also measure the stabilized power output (SPO) near the maximum power point for these two perovskite cells (Supplementary Fig. 2). The SPOs are about $17.69 \%$ and $13.1 \%$ for cells based on large and small grains, respectively. In addition, energy-dispersive Xray spectroscopy (EDS) measurement (Supplementary Fig. 3) also suggests that there is no clear correlation between the $\mathrm{Pb}$ and I distribution and the grain morphology (e.g., grain vs. grain boundary) in both samples. $\mathrm{Pb}$ and I stay roughly constant across multiple grains within experimental noise and the ratio of $\mathrm{I} / \mathrm{Pb}$ is essentially the same for both samples. Consistent with previous studies ${ }^{23-25}$, uncapped $\mathrm{MAPbI}_{3}$ thin films degrade within several hours under the ambient light and humidity conditions, as shown in Supplementary Fig. 4. To prevent such rapid degradation, we have capped the sample surface with a thin ( $30 \mathrm{~nm}$ thick) layer of spin-coated PMMA ${ }^{47}$ in the following experiments. As shown in the schematic in Fig. 1a, part of the $\mathrm{PMMA}_{\mathrm{MAPbI}}$ films is scratched away to expose the glass substrate as the reference region for the MIM imaging.

Before discussing our main results, it is instructive to review the contrast mechanism and response function of the MIM technique. Figure $1 \mathrm{~b}$ shows the finite-element analysis (FEA) results based on the actual tip/sample geometry and material properties. Details of the FEA process are described in Supplementary Fig. 5. As the conductivity $\sigma$ of $\mathrm{MAPbI}_{3}$ increases, the microwave electrical fields are gradually pushed away from this layer due to the screening effect from free carriers. As a result, the MIM-Im signal, which is proportional to the tip-sample capacitance, starts to rise as $\sigma>10^{-1} \mathrm{~S} \mathrm{~m}^{-1}$, increases monotonically as a function of $\sigma$, and saturates for $\sigma>10^{3} \mathrm{~S} \mathrm{~m}^{-1}$. On the other hand, the MIM-Re signal (proportional to the electrical loss at $1 \mathrm{GHz}$ ), peaks around $\sigma=10^{1} \mathrm{~S} \mathrm{~m}^{-1}$ and decreases for both higher and lower conductivities. Note that while the simulation is performed for a uniform $\mathrm{MAPbI}_{3}$ layer, the span of the quasistatic electric field, which determines the lateral resolution and vertical probing depth, is set by the tip diameter. The MIM thus maps out the local variation of conductivity in the mesoscopic length scale of around $100 \mathrm{~nm}$.

Figure $2 \mathrm{a}, \mathrm{b}$ displays the AFM and MIM images of the samples with $18 \%$ and $15 \%$ PCEs, respectively. The large difference in grain sizes between these two films is evident from the topographic data. Without the illumination, there is little MIM contrast between the highly resistive perovskite film and the glass substrate (Supplementary Fig. 6). When illuminated by an abovegap continuous-wave $532 \mathrm{~nm}$ laser with a power intensity $P$ of $100 \mathrm{~mW} \mathrm{~cm}^{-2}$ (on the order of 1 Sun at AM 1.5), the $18 \%$ PCE sample exhibits clear photo-induced MIM signals, while the effect is much weaker on the $15 \%$ PCE sample. As a control experiment, we have also performed the MIM imaging when the $18 \%$ PCE sample is illuminated by a below-gap $980-\mathrm{nm}$ laser with the same power density of $100 \mathrm{~mW} \mathrm{~cm}^{-2}$, as shown in Supplementary Fig. 6. The absence of photo-induced MIM signals with belowgap illumination further confirms the photoconductivity in the $\mathrm{MAPbI}_{3}$ films. a
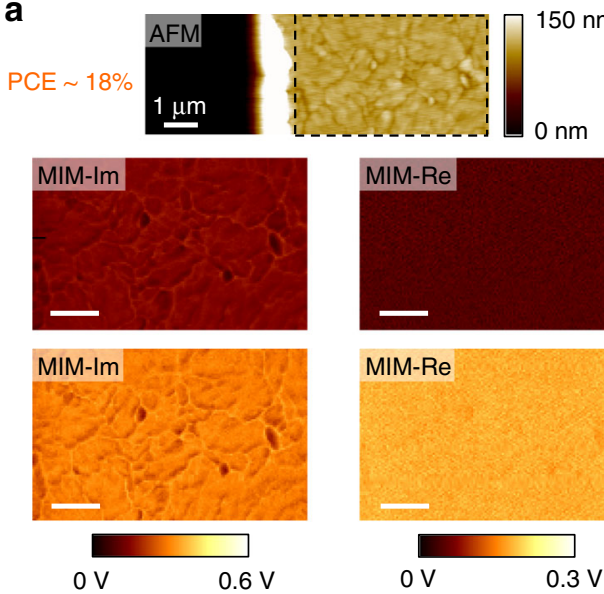
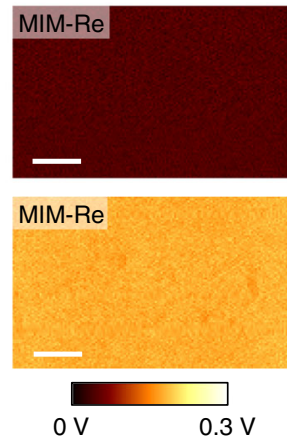

b

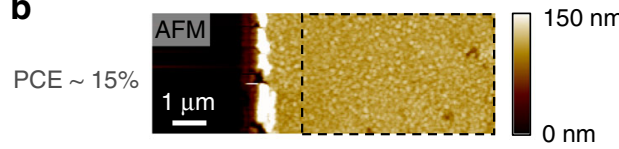

Laser off
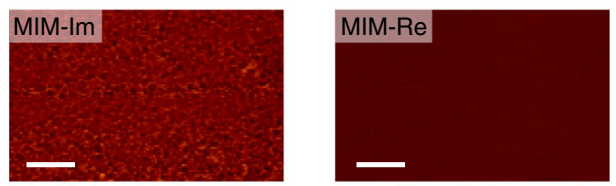

Laser on $100 \mathrm{~mW} \mathrm{~cm}^{-2}$
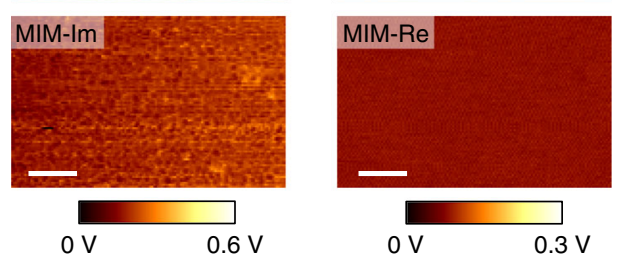

Fig. 2 Nanoscale photoconductivity imaging on MAPbl 3 . a AFM (top) and MIM-Im/Re images (inside the black dashed rectangles in the AFM data) when the 532-nm laser is turned off (middle) and on (bottom) for the 18\% PCE sample. The same results for the 15\% PCE sample are shown in $\mathbf{b}$. The scale bars in $\mathbf{a}$ and $\mathbf{b}$ are $1 \mu \mathrm{m}$ 

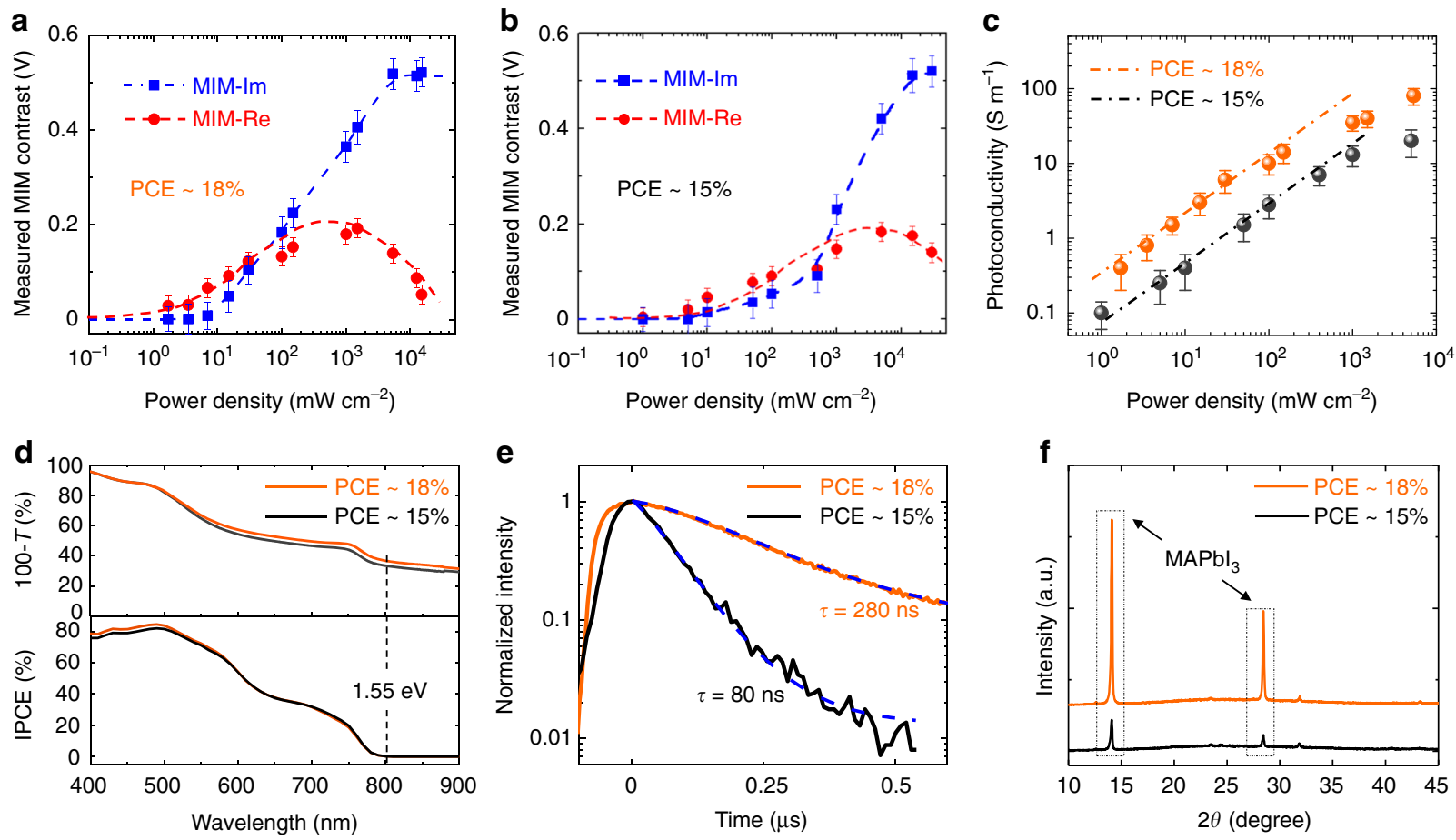

Fig. 3 Photoconductivity and other characterizations of $\mathrm{MAPb}_{3}$ thin films. a Measured MIM signals of the PCE around 18\% and $\mathbf{b} 15 \%$ samples (with respect to the values without light) as a function of the incident $532 \mathrm{~nm}$-laser power. The raw data of $\mathbf{a}$ and $\mathbf{b}$ are shown in Supplementary Fig. 5 . The error bars are estimated from the standard deviation of the raw MIM data. c Photoconductivity of both samples extracted from the experimental data vs. the laser intensity. The dash-dotted lines are linear fits for laser power below $100 \mathrm{~mW} \mathrm{~cm}^{-2}$. d Optical absorptivity (upper panel) and IPCE (low panel) spectra of the perovskite thin films with $18 \%$ and $15 \%$ PCE. The optical gap of $\mathrm{MAPbl}_{3}$ is indicated in the plots. e TRPL measurements of the two samples, from which the carrier lifetime can be extracted. The excitation power in the TRPL measurements is around $30 \mathrm{~mW} \mathrm{~cm}^{-2}$, which is within the linear regime of the MIM data in c. $\mathbf{f}$ XRD measurements of the two samples, indicating a much higher crystallinity of the $18 \%$ PCE sample

Quantitative analysis of photo-response. The intrinsic photoconductivity images in Fig. 2 contain much information on the photon-to-electron conversion process, which is at the heart of solar cell devices. For the quantitative analysis, we first focus on the power dependence of MIM response on the 18\% PCE sample. Figure 3a shows the average MIM signals over an area of $3.5 \times 3.5$ $\mu \mathrm{m}$ as a function of the $532 \mathrm{~nm}$-laser intensity (raw data displayed in Supplementary Fig. 7a). The MIM-Im signals increase monotonically with the laser power and saturate for both $P<10 \mathrm{~mW}$ $\mathrm{cm}^{-2}$ and $P>10^{4} \mathrm{~mW} \mathrm{~cm}{ }^{-2}$. The MIM-Re signals, on the other hand, reach a peak at $P$ around $10^{3} \mathrm{~mW} \mathrm{~cm}^{-2}$ and decrease on both sides. The close resemblance between Figs. $1 \mathrm{~b}$ and $3 \mathrm{a}$ allows us to quantitatively extract $\sigma$ from the measured data. Since the film thickness is comparable to the tip diameter, the MIM is measuring the effective photoconductivity $\sigma$ averaged over the vertical direction, which can be derived as follows ${ }^{48}$ (assuming complete absorption by the film):

$$
\sigma=\frac{\eta}{H}\left(\frac{P \tau_{e}}{h \nu} \cdot e \cdot \mu_{e}+\frac{P \tau_{h}}{h \nu} \cdot e \cdot \mu_{h}\right)
$$

As an order-of-magnitude estimate, i.e., taking the incident photon-to-electron conversion efficiency (IPCE, hereafter denoted as $\eta) \eta=1$, film thickness $H=100 \mathrm{~nm}$, electron and hole lifetime $\tau_{e} \approx \tau_{h} \approx 10^{2} \mathrm{~ns}$, photon energy $h \nu=2.33 \mathrm{eV}$, electron and hole mobility $\mu_{e} \approx \mu_{h} \approx 10^{2} \mathrm{~cm}^{2} \mathrm{~V}^{-1} \mathrm{~s}^{-1}$, we obtain $\sigma \approx 10 \mathrm{~S} \mathrm{~m}^{-1}$ at $P=100 \mathrm{~mW} \mathrm{~cm}^{-2}$, which is in excellent agreement with the data in Fig. 3c. The corresponding electron (hole) density is $n(p)=P \tau_{e}$ $(h) /(H \cdot h \nu) \approx 3 \times 10^{15} \mathrm{~cm}^{-3}$, consistent with other investigations ${ }^{49}$. For laser intensity above $100 \mathrm{~mW} \mathrm{~cm}^{-2}$, the sub-linear power dependence observed in Fig. $3 \mathrm{c}$ may result from the saturation of photo-generated carriers ${ }^{50}$. On the other hand, it is generally believed that trap states in $\mathrm{MAPbI}_{3}$ remain unfilled at around 1 Sun and become filled up to 10-100 Sun under the steady-state illumination $^{44}$. It is thus possible that trap states also play a role in the saturation of photoconductivity in Fig. 3c.

We now turn to the comparison between the two $\mathrm{MAPbI}_{3}$ thin films. Following the same procedure, the MIM signals and powerdependent photoconductivity of the 15\% PCE sample are (raw data shown in Supplementary Fig. 7b) plotted in Fig. 3b, c, respectively. In the regime relevant to practical solar cell applications ( $P$ no greater than $100 \mathrm{~mW} \mathrm{~cm}^{-2}$ ), the photoconductivity of the $18 \%$ PCE sample is five to six times higher than that of the 15\% PCE sample. The two films show very similar wavelength-dependent absorptivity and IPCE over the visible spectrum, as seen in Fig. 3d. The time-resolved photoluminescence (TRPL) data in Fig. 3e show that the total electron-hole recombination time is $280 \mathrm{~ns}$ and $80 \mathrm{~ns}$ for the $18 \%$ and $15 \%$ PCE samples, respectively, from which we estimate that the average carrier lifetime differs by a factor of three between the two films. As a result, the average mobility is also improved by a considerable amount (about two times) in the $18 \%$ film. Finally, from our X-ray diffraction (XRD) data in Fig. 3f, the substantially better crystallinity of the $18 \%$ film is vividly demonstrated by its much stronger $\mathrm{MAPbI}_{3}$ peak than the $15 \%$ counterpart. In all, as the average grain size increases from a few hundred nanometers to a few micrometers due to the better growth control, both the electron-hole recombination rate and transport scattering rate are considerably reduced.

Photoconductivity of grain boundaries. To understand the role of GBs, we directly compare the local photoconductivity between grains and GBs in the $18 \%$ PCE sample. From the AFM image in Fig. 4a, appreciable inhomogeneity is seen on the polycrystalline 
a
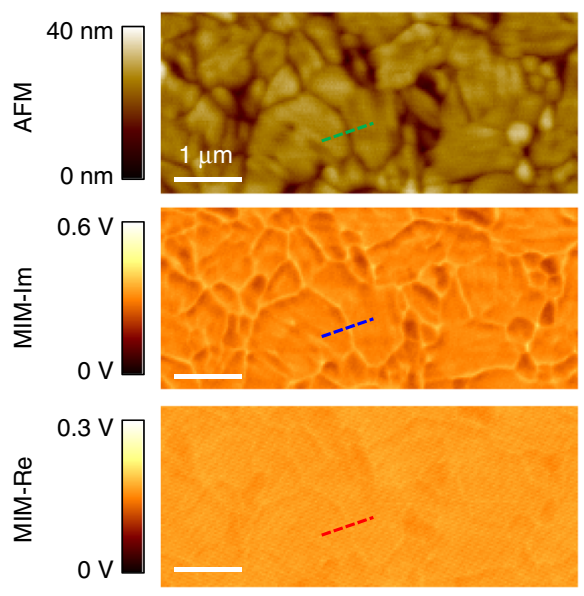

b

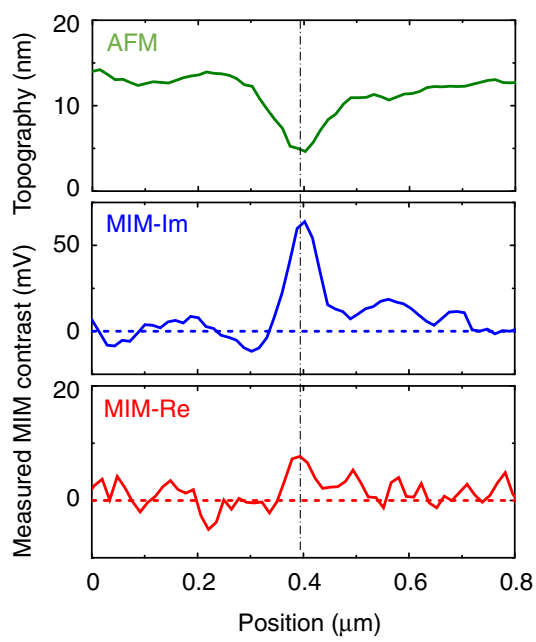

c
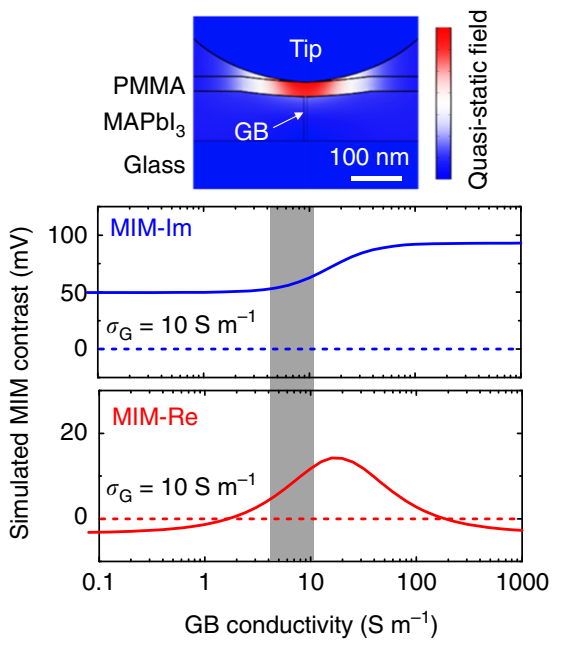

Fig. 4 Various microstructures on the perovskite film. a AFM and MIM images showing the detailed features on the $M A P b I_{3}$ thin film under $100 \mathrm{~mW} \mathrm{~cm}^{-2}$ illumination of the $532 \mathrm{~nm}$ laser. The photoresponse is uniform for grains with different heights and lateral sizes. All scale bars are $1 \mu \mathrm{m}$. $\mathbf{b}$ Line profiles across a single grain boundary (green in AFM, blue in MIM-Im, and red in MIM-Re) in a. The MIM contrast signals are referenced to the mean values on

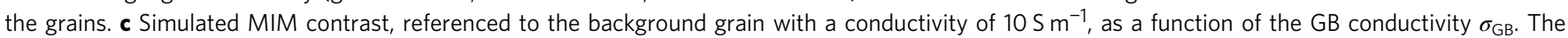
shaded column corresponds to the measured data within the experimental errors. The inset shows the simulated quasi-static displacement-field distribution for $\sigma_{\mathrm{G}}=\sigma_{\mathrm{GB}}=10 \mathrm{~S} \mathrm{~m}^{-1}$

$\mathrm{MAPbI}_{3}$ thin film, with grain sizes ranging from sub-micrometer to a few micrometers and GBs appearing as trenches of about 10 $\mathrm{nm}$ in depth. In contrast, the corresponding MIM images are relatively uniform over grains with different heights and lateral sizes. In Fig. $4 \mathrm{~b}$, we compare the line profiles across a single GB from the AFM and MIM images. Note that the MIM-Im channel is susceptible to the topographic crosstalk, while the MIM-Re signal is less sensitive to the surface roughness. We emphasize that the topographic mixing is not proportional to the AFM data and cannot be removed by simple image processing. The AFM data represent the position of the tip apex when scanning on a surface with certain roughness. The topographic mixing in the MIM-Im data, on the other hand, is mostly due to the stray capacitance from the non-apex part when the tip tracks the sample surface. For quantitative analysis of the GB photoconductivity $\sigma_{\mathrm{GB}}$, we perform the $3 \mathrm{D}$ FEA simulation (detailed in Supplementary Fig. 8), in which the GB is modeled as a thin slab $(10 \mathrm{~nm}$ in width $w)$ with a height difference $\Delta H$ of $10 \mathrm{~nm}$ lower than the grains $\left(10 \mathrm{~S} \mathrm{~m}^{-1}\right)$. Within the experimental uncertainty, a comparison between the MIM data and the FEA shows that $\sigma_{\mathrm{GB}}$ is within a factor of two from $\sigma_{\mathrm{G}}$. As a control experiment, we have also conducted the MIM measurement on a thicker (300 $\mathrm{nm}$ ) film of the $18 \%$ PCE sample and observed the same results (Supplementary Fig. 9), which indicates that the MIM signals are not dominated by the possible surface recombination in the 100 $\mathrm{nm}$ film. The result of nearly uniform photoconductivity is striking since GBs contain a large amount of dangling bonds, which usually lead to trap states detrimental to carrier generation and charge transport in conventional solar cell materials ${ }^{7}$. For halide perovskites, the role of GBs has been under debate over the years. While some theoretical studies showed that GBs only create shallow in-gap states due to the high ionicity and strong $\mathrm{Pb}-\mathrm{I}$ anti-bonding ${ }^{15,16}$, others indicated that GBs are the major recombination centers and should be passivated ${ }^{22}$. Our measurements provide direct experimental evidence that, at least for encapsulated thin films with micrometer-sized grains, GBs are relatively benign to carrier generation and transport. Perovskite films with better crystallinity, on the other hand, should be the direction to further improve the efficiency of PSCs.
Spatial evolution of the degradation process. The air stability of the perovskite films, which is arguably the most serious bottleneck towards commercial applications ${ }^{23-29}$, has also been studied by the MIM. Figure 5a shows selected AFM and MIM images of a PMMA-coated $\mathrm{MAPbI}_{3}$ film over 1 week under the ambient condition with a relative humidity of $35 \%$ at $23^{\circ} \mathrm{C}$ (complete set of data included in Supplementary Fig. 10). Little change in the photoconductivity is observed on the first day or two, suggesting that the PMMA capping layer was initially effective in preventing the film degradation. Starting from Day 3, however, appreciable reduction of the photo-response is seen in certain regions of the film. Interestingly, the degradation does not emerge from GBs but is associated with the disintegration of large grains, which substantially reduces the photoconductivity in the surrounding regions. The degraded areas continue to grow during the subsequent days and the photoconductivity completely diminishes after Day 6. We note that recent transmission electron microscopy (TEM) studies have revealed the existence of twin boundaries that are not seen in traditional SEM or AFM images $^{51}$. Our results are thus only valid for the GBs with clear topographic features. As a control experiment, we also continuously illuminate another sample for $9 \mathrm{~h}$ (same as the total imaging time in the aging experiment above) and observe no change in both topographic and photoconductivity maps (Supplementary Fig. 11). The illumination by itself, therefore, is not sufficient to cause the drastic suppression of photoconductivity in Fig. 5a.

To gain further insight on the aging process, we have carried out structural and optical characterizations on films prepared by the same method and exposed to the same conditions. As seen in the XRD results in Fig. $5 \mathrm{~b}$, the characteristic $\mathrm{PbI}_{2}$ peak at $2 \theta=$ $12.66^{\circ}$ appears on Day 1 and becomes significant on Day 3. The spectrum on Day 6 is dominated by the strong $\mathrm{PbI}_{2}$ peak, indicative of the nearly complete decomposition of $\mathrm{MAPbI}_{3}$ after 1 week. Note that the emergence of $\mathrm{PbI}_{2}$ reduces the local photoconductivity because its absorption edge at $525 \mathrm{~nm}^{52}$ is at higher energy than our $532 \mathrm{~nm}$ laser. The TRPL data plotted in Fig. $5 c$ also demonstrate the same trend. While little change is observed after one day, the absolute PL intensity drops 
a
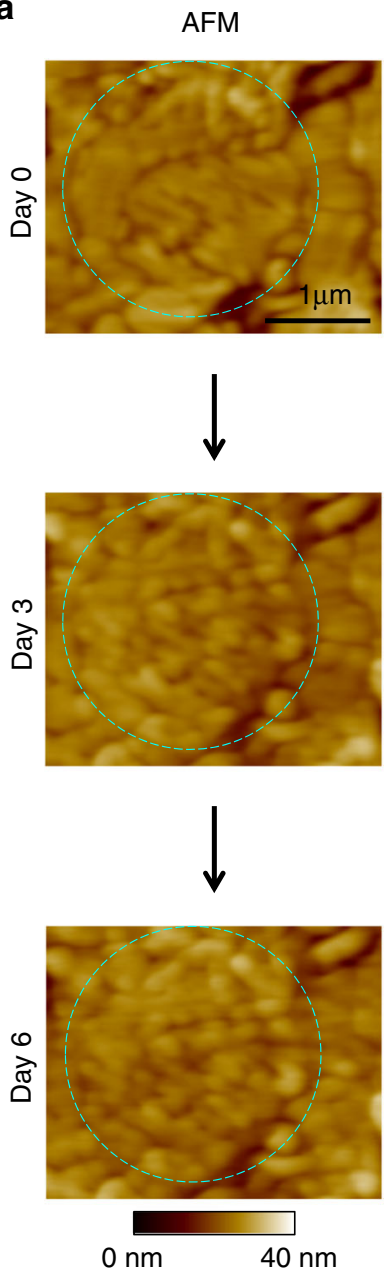

MIM-Im

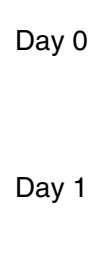

Day 2.3

Day 2.7

Day 3.1

Day 4

Day 5

Day 6
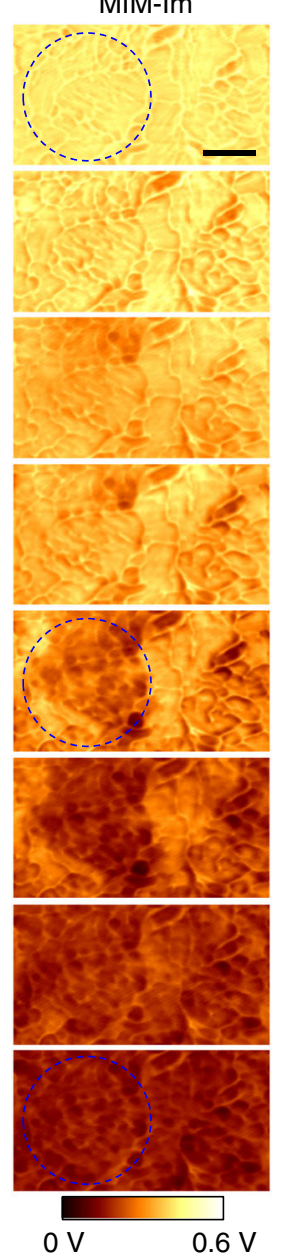

MIM-Re
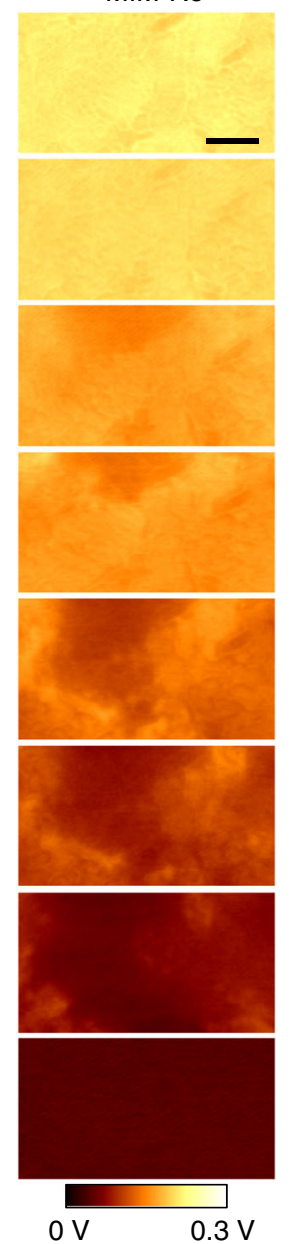

b

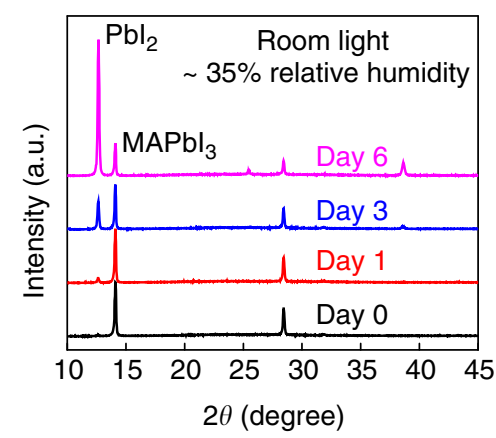

C

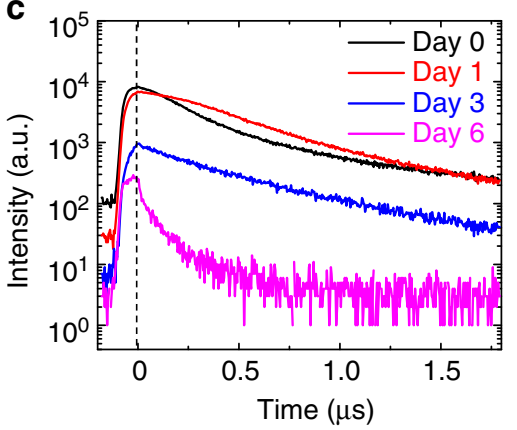

d

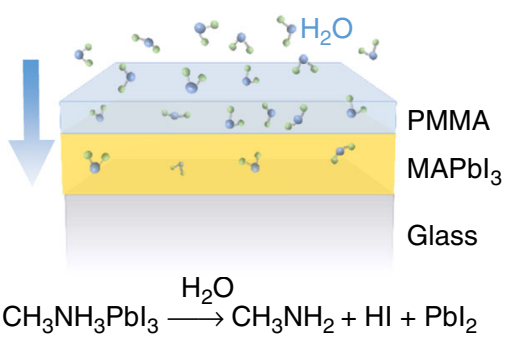

Fig. 5 Degradation process of the $\mathrm{MAPbl}_{3}$ thin film. a MIM images over 1 week with $35 \%$ relative humidity at $23^{\circ} \mathrm{C}$. Zoom-in AFM images on Days 0,3 , and 6 are shown to the left. Note that the fine features on large grains on Day 0 (inside dashed circles) are associated with crystal edges or terraces, which are common for polycrystalline thin films. The sample was only illuminated by the 532-nm laser during the MIM imaging for about 30 min at each frame. All scale bars are $1 \mu \mathrm{m}$. $\mathbf{b}$ Selected XRD and $\mathbf{c}$ TRPL data on MAPbl ${ }_{3}$ films prepared by the same method and exposed to the same conditions. The XRD peak at $2 \theta=12.66^{\circ}$ is associated with $\mathrm{Pbl}_{2}$. $\mathbf{d}$ Schematic of the aging process due to the diffusion of water molecules through the PMMA layer, which drives the decomposition of $\mathrm{MAPbl}_{3}$ into $\mathrm{CH}_{3} \mathrm{NH}_{2}$ and $\mathrm{HI}$ in the gas phase and $\mathrm{Pbl}_{2}$ in the solid phase

substantially on Day 3 and the TRPL decay rate increases rapidly on Day 6. Interestingly, the TRPL decay rates are similar for the first three days, suggesting that the carrier lifetime remains large in the unaffected regions. Combining the microscopic and macroscopic results, we conclude that the deterioration of the encapsulated film is initiated and accelerated by the water molecules slowly diffused through the PMMA layer, which drive the decomposition of large $\mathrm{MAPbI}_{3}$ grains into $\mathrm{PbI}_{2}$ in the solid phase and $\mathrm{CH}_{3} \mathrm{NH}_{2}$ and $\mathrm{HI}$ in the gas phase ${ }^{23-26}$. Again, GBs are not the nucleation centers during the degradation.

\section{Discussion}

To summarize, we report the intrinsic photoconductivity mapping of thin-film $\mathrm{MAPbI}_{3}$ with different PCEs under above-gap illuminations. The large photoconductivity of the higher efficiency sample is a direct consequence of the high carrier mobility and long lifetime because of the improved crystallinity. Surprisingly, the GBs exhibit photo-responses comparable to the grains, and they are not the nucleation centers for the degradation process. Our results highlight the unique defect structures responsible for the remarkable performance of PSC devices, and address the significance of crystallinity to further improve their energy conversion efficiency. We also emphasize that the nanoscale photoconductivity imaging by microwave microscopy represents a new methodology in optoelectronic research. With future development to incorporate broadband illumination, variable temperatures, and humidity-controlled environment, we expect to obtain further insights on the intriguing photo physics and air sensitivity of the hybrid perovskite materials.

\section{Methods}

Preparation of $\mathbf{M A P b l}_{\mathbf{3}}$ perovskite thin films. The perovskite films in this work were deposited on top of cover glasses using the stoichiometry and nonstoichiometry solvent-solvent extraction method ${ }^{21}$. In short, $30 \mathrm{wt} \%$ methylammonium iodide (MAI) and lead iodide $\left(\mathrm{PbI}_{2}\right)\left(\mathrm{MAI} / \mathrm{PbI}_{2}=1 / 1, \mathrm{MAI} / \mathrm{PbI}_{2}=1.2 /\right.$ 1) were dissolved in a mixed solvent of 1-N-methyl-2-pyrrolidinone (NMP) and $\gamma$ butylrolactone $(\mathrm{GBL})(\mathrm{NMP} / \mathrm{GBL}=7 / 3$ weight ratio). The cover glass with $80 \mu \mathrm{l}$ precursor solution was spun at $6000 \mathrm{rpm}$ for $25 \mathrm{~s}$, and immediately dipped into a vigorously stirred diethyl ether bath for $1 \mathrm{~min}$. The perovskite films rapidly crystallized during the bathing process and were further annealed (stoichiometry precursor: $100^{\circ} \mathrm{C}$ for $10 \mathrm{~min}$; non-stoichiometry precursor: $150^{\circ} \mathrm{C}$ for $15 \mathrm{~min}$ ) with a petri-dish covered to remove excess organic salt. Within the experimental errors of our characterization tools, these two types of perovskite films show the same $\mathrm{MAPbI}_{3}$ perovskite composition and crystal structure and only differ in grain size and crystallinity ${ }^{21}$. The encapsulated perovskite films were capped with PMMA $\left(M_{w}\right.$ about 120,000$)$ film by spin-coating $15 \mathrm{mg} \mathrm{ml}^{-1}$ PMMA in chlorobenzene solution at $4000 \mathrm{rpm}$ for $35 \mathrm{~s}$. 
Structural and optical characterizations. The absorption spectra were measured by a UV/Vis spectrometer (Cary-6000i). The perovskite structure was characterized by an X-ray diffractometer (Rigaku D/Max 2200) using the $\mathrm{Cu} \mathrm{K} \alpha$ radiation. The incident photon-to-electron conversion efficiency (IPCE) experiment was carried out in a solar cell quantum efficiency measurement system (QEX10, PV Measurements). The time-resolved photoluminescence (TRPL) measurements were conducted in a home-built time correlated single photon counting system, where light source is a Fianium Supercontinuum high power broadband fiber laser (SC400-2-PP). The excitation wavelength was $500 \mathrm{~nm}$ with a spot area of $300 \times 300$ $\mu \mathrm{m}$, a power of about $5 \mu \mathrm{W}$, and a repetition rate of $0.1 \mathrm{MHz}$.

Microwave impedance microscopy and finite-element analysis. The MIM in this work is based on a modified ParkAFM XE-100 platform with bottom illumination. The customized shielded cantilever probes ${ }^{40}$ are commercially available from PrimeNano Inc.

Finite-element analysis is performed using the AC/DC module of commercial software COMSOL4.4. Supplementary Figure 5 shows the FEA simulation result using the tip/sample geometry and material properties ${ }^{39}$. The upper part of the tip is modeled as a truncated cone with a half-angle of $15^{\circ}$ and a diameter of $200 \mathrm{~nm}$ at the bottom surface. The radius of curvature of the tip apex (modeled as a truncated sphere), $250 \mathrm{~nm}$ in this case, is determined by calibration on standard samples. The relative dielectric constant $\varepsilon$ of $\mathrm{MAPbI}_{3}$ is assumed to be 60 , consistent with that reported in the literature ${ }^{7}$. From the AFM data, the thicknesses of the PMMA $(\varepsilon=$ 3, http://webhotel2.tut.fi/projects/caeds/tekstit/plastics/plastics_PMMA.pdf) and $\mathrm{MAPbI}_{3}$ are $30 \mathrm{~nm}$ and $100 \mathrm{~nm}$, respectively. Supplementary Figure 5a shows the simulated real and imaginary parts of the tip-sample admittance as a function of the conductivity of the $\mathrm{MAPbI}_{3}$ layer, using the values at $\sigma=0$ as the reference points. The MIM electronics is calibrated in that an admittance contrast of $1 \mathrm{nS}$ corresponds to an output voltage of $3.5 \mathrm{mV}$. In general, the MIM-Im signal, which is proportional to the tip-sample capacitance, increases monotonically as a function of $\sigma$. The MIM-Re signal, which corresponds to the electrical loss at 1 $\mathrm{GHz}$, peaks at intermediate $\sigma$ and decreases for both good conductors and good insulators. The quasi-static potential and displacement field distributions around the tip-sample junction are shown in Supplementary Fig. $5 \mathrm{~b}$ for three characteristic conductivities at $0.1,10$, and $1000 \mathrm{~S} \mathrm{~m}^{-1}$, respectively.

The 2D axisymmetric simulation in Supplementary Fig. 5 does not work for the modeling of grain boundaries (GBs) because of the lack of rotational symmetry. To quantitatively understand the experimental data, we have performed $3 \mathrm{D}$ simulation and modeled the GB as a thin slab sandwiched between two adjacent grains. The center of the GB is $10 \mathrm{~nm}(\Delta H)$ lower than the surrounding grains, giving rise to the topographic artifact to the MIM signals described below. The width $(w)$ of the $\mathrm{GB}$, within which the electronic conductivity may differ from $\sigma_{\mathrm{G}}$ due to dangling bonds and other defects, is assumed to be $10 \mathrm{~nm}$. The simulated MIM signals when the tip locates on top of the GB are plotted in Supplementary Fig. 8a, together with the signals when the tip locates on top of the grains $\left(\sigma_{\mathrm{G}}=10 \mathrm{~S} \mathrm{~m}^{-1}\right.$ under the 100 $\mathrm{mW} \mathrm{cm}{ }^{-2}$ illumination). The tip-sample interaction at various $\sigma_{\mathrm{GB}}$ 's can be visualized by the quasi-static displacement field distribution in Supplementary Fig. 8b. The relative MIM contrast (GBs vs. grains) in our experiment corresponds to the difference between solid and dashed lines in Supplementary Fig. 8a. Note that the MIM-Im contrast is considerably affected by the topographic crosstalk, as the GB signals are always higher than that within the grains for all $\sigma_{\mathrm{GB}}$ 's. Nevertheless, by comparing the FEA results in Fig. $4 \mathrm{c}$ and the experimental data $(60 \mathrm{mV}$ contrast in MIM-Im and $10 \mathrm{mV}$ contrast in MIM-Re) in Fig. 4b, it is evident that $\sigma_{\mathrm{GB}}$ is not substantially smaller than $\sigma_{\mathrm{G}}$ (within a factor of 2). We therefore conclude that $\sigma_{\mathrm{GB}}$ is not substantially smaller than $\sigma_{\mathrm{G}}$ (within a factor of 2). We therefore conclude that GBs are intrinsically benign in $\mathrm{MAPbI}_{3}$ thin films with photoconductivity comparable to that within the grains ${ }^{15}$.

Data availability. The data that support the findings of this work are available from the corresponding author upon reasonable request.

Received: 18 April 2017 Accepted: 21 November 2017

Published online: 20 December 2017

\section{References}

1. Kojima, A., Teshima, K., Shirai, Y. \& Miyasaka, T. Organometal halide perovskites as visible-light sensitizers for photovoltaic cells. J. Am. Chem. Soc. 131, 6050-6051 (2009).

2. Lee, M. M., Teuscher, J., Miyasaka, T., Murakami, T. N. \& Snaith, H. J. Efficient hybrid solar cells based on meso-superstructured organometal halide perovskites. Science 338, 643-647 (2012).

3. Burschka, J. et al. Sequential deposition as a route to high-performance perovskite-sensitized solar cells. Nature 499, 316-319 (2013).

4. Liu, M., Johnston, M. B. \& Snaith, H. J. Efficient planar heterojunction perovskite solar cells by vapour deposition. Nature 501, 395-398 (2013).
5. Zhou, H. et al. Interface engineering of highly efficient perovskite solar cells. Science 345, 542-546 (2014).

6. Nie, W. et al. High-efficiency solution-processed perovskite solar cells with millimeter-scale grains. Science 347, 522-525 (2015).

7. Green, M. A., Ho-Baillie, A. \& Snaith, H. J. The emergence of perovskite solar cells. Nat. Photonics 8, 506-514 (2014).

8. Berry, J. et al. Hybrid origanic-inorganic perovskites (HOIPs): opportunities and challenges. Adv. Mater. 27, 5102-5112 (2015).

9. Stranks, S. D. \& Snaith, H. J. Metal-halide perovskite for photovoltaic and lightemitting devices. Nat. Nanotechnol. 10, 391-402 (2015).

10. Lewis, N. S. Research opportunities to advance solar energy utilization. Science 351, 1920 (2016)

11. Ponseca, C. S. et al. Organometal halide perovskite solar cell materials rationalized: ultrafast charge generation, high and microsecond-long balanced mobilities, and slow recombination. J. Am. Chem. Soc. 136, 5189-5190 (2014).

12. Oga, H., Saeki, A., Ogomi, Y., Hayase, S. \& Seki, S. Improved understanding of the electronic and energetic landscapes of perovskite solar cells: high local charge carrier mobility, reduced recombination, and extremely shallow traps. J. Am. Chem. Soc. 136, 13818-13825 (2014).

13. Stranks, S. D. et al. Electron-hole diffusion lengths exceeding 1 micrometer in an organometal trihalide perovskite absorber. Science 342, 341-344 (2013).

14. Shi, D. et al. Low trap-state density and long carrier diffusion in organolead trihalide perovskite single crystals. Science 347, 519-522 (2015).

15. Yin, W. J., Shi, T. \& Yan, Y. Unique properties of halide perovskites as possible origins of the superior solar cell performance. Adv. Mater. 26, 4653-4658 (2014).

16. Yin, W. J., Shi, T. \& Yan, Y. Unusual defect physics in $\mathrm{CH}_{3} \mathrm{NH}_{3} \mathrm{PbI}_{3}$ perovskite solar cell absorber. Appl. Phys. Lett. 104, 063903 (2014).

17. Xiao, Z. G. et al. Solvent annealing of perovskite-induced crystal growth for photovoltaic-device efficiency enhancement. Adv. Mater. 26, 6503-6509 (2014).

18. Yang, B. et al. Perovskite solar cells with near $100 \%$ internal quantum efficiency based on large single crystalline grains and vertical bulk heterojunctions. J. Am. Chem. Soc. 137, 9210-9213 (2015).

19. De Marco, N. et al. Guanidinium: a route to enhanced carrier lifetime and open-circuit voltage in hybrid perovskite solar cells. Nano Lett. 16, 1009-1016 (2016).

20. Zhang, W. et al. Enhanced optoelectronic quality of perovskite thin films with hypophosphorous acid for planar heterojunction solar cells. Nat. Commun. 6 10030 (2015)

21. Yang, M., Zhou, Y., Zeng, Y., Jiang, C. S. \& Padture, N. P. et al Squarecentimeter solution-processed planar $\mathrm{CH} 3 \mathrm{NH} 3 \mathrm{PbI} 3$ perovskite solar cells with efficiency exceeding 15\%. Adv. Mater. 27, 6363-6370 (2015).

22. Long, R., Liu, J. \& Prezhdo, O. V. Unravelling the effects of grain boundary and chemical doping on electron-hole recombination in $\mathrm{CH}_{3} \mathrm{NH}_{3} \mathrm{PbI}_{3}$ Perovskite by Time-Domain Atomistic Simulation. J. Am. Chem. Soc. 138, 3884-3890 (2016).

23. Niu, G. et al. Study on the stability of $\mathrm{CH} 3 \mathrm{NH} 3 \mathrm{PbI} 3$ films and the effect of postmodification by aluminum oxide in all-solid-state hybrid solar cells. J. Mater. Chem. A 2, 705-710 (2014).

24. Leijtens, T. et al. Stability of metal halide perovskite solar cells. Adv. Energy Mater. 5, 1500963 (2015)

25. Han, Y. et al. Degradation observations of encapsulated planar CH3NH3PbI3 perovskite solar cells at high temperatures and humidity. J. Mater. Chem. A 3 , 8139-8147 (2015).

26. Niu, G., Guo, X. \& Wang, L. Review of recent progress in chemical stability of perovskite solar cells. J. Mater. Chem. A 3, 8970-8980 (2014).

27. Berhe, T. A. et al. Organometal halide perovskite solar cells: degradation and stability. Energy Environ. Sci. 9, 323-356 (2016).

28. Yang, J., Siempelkamp, B. D., Liu, D. \& Kelly, T. L. Investigation of $\mathrm{CH} 3 \mathrm{NH} 3 \mathrm{PbI} 3$ degradation rates and mechanisms in controlled humidity environments using in situ techniques. ACS Nano 9, 1955-1963 (2015).

29. Divitini, G. et al. In situ observation of heat-induced degradation of perovskite solar cells. Nat. Energy 1, 15012 (2016).

30. Jiang, C. et al. Carrier separation and transport in perovskite solar cells studied by nanometre-scale profiling of electrical potential. Nat. Commun. 6, 8397 (2015).

31. Xiao, Z. et al. Giant switchable photovoltaic effect in organometal trihalide perovskite devices. Nat. Mater. 14, 193-198 (2014).

32. Zhao, Z., Chen, X., Wu, H., Wu, X. \& Cao, G. Probing the photovoltage and photocurrent in perovskite solar cells with nanoscale resolution. Adv. Funct. Mater. 26, 3048-3058 (2016)

33. Yun, J. S. et al. Benefit of grain boundaries in organic-inorganic halide planar perovskite solar cells. J. Phys. Chem. Lett. 6, 875-880 (2015).

34. Kutes, Y. et al. Mapping the photoresponse of $\mathrm{CH}_{3} \mathrm{NH}_{3} \mathrm{PbI}_{3}$ hybrid perovskite thin films at the nanoscale. Nano Lett. 16, 3434-3441 (2016). 
35. Son, D. et al. Self-formed grain boundary healing layer for highly efficient $\mathrm{CH}_{3} \mathrm{NH}_{3} \mathrm{PbI}_{3}$ perovskite solar cells. Nat. Energy 1, 16081 (2016).

36. Kutes, Y. et al. Direct observation of ferroelectric domains in solutionprocessed $\mathrm{CH} 3 \mathrm{NH} 3 \mathrm{PbI} 3$ perovskite thin films. J. Phys. Chem. Lett. 5, 3335-3339 (2014).

37. Fan, Z. et al. Ferroelectricity of $\mathrm{CH}_{3} \mathrm{NH}_{3} \mathrm{PbI}_{3}$ perovskite. J. Phys. Chem. Lett. 6 , 1155-1161 (2015)

38. deQuilettes, D. W. et al. Impact of microstructure on local carrier lifetime in perovskite solar cells. Science 348, 683-686 (2015).

39. Lai, K., Kundhikanjana, W., Kelly, M. \& Shen, Z. X. Modeling and characterization of a cantilever-based near-field scanning microwave impedance microscope. Rev. Sci. Instrum. 79, 063703 (2008).

40. Lai, K., Kundhikanjana, W., Kelly, M. A. \& Shen, Z. X. Nanoscale microwave microscopy using shielded cantilever probes. Appl. Nanosci. 1, 13-18 (2011).

41. Tuteja, M., Koirala, P., Maclaren, S., Collins, R. \& Rockett, A. Direct observation of electrical properties of grain boundaries in sputter-deposited $\mathrm{CdTe}$ using scan-probe microwave reflectivity based capacitance measurements. Appl. Phys. Lett. 107, 142106 (2015).

42. Yang, Y. et al. Batch-fabricated cantilever probes with electrical shielding for nanoscale dielectric and conductivity imaging. J. Micromech. Microeng. 22 115040 (2012)

43. Marchioro, A. et al. Unravelling the mechanism of photoinduced charge transfer processes in lead iodide perovskite solar cells. Nat. Photonics $\mathbf{8}$, 250-255 (2014).

44. Hutter, E. M., Eperon, G. E., Stranks, S. D. \& Savenije, T. J. Charge carriers in planar and meso-structured organic-inorganic perovskites: mobilities, lifetimes, and concentrations of trap states. J. Phys. Chem. Lett. 6, 3082-3090 (2015).

45. Reid, O. G., Yang, M., Kopidakis, N., Zhu, K. \& Rumbles, G. Grain-size-limited mobility in methylammonium lead iodide perovskite thin films. ACS Energy Lett. 1, 561-565 (2016).

46. Ihly, R. et al. Efficient charge extraction and slow recombination in organic-inorganic perovskites capped with semiconducting single-walled carbon nanotubes. Energy Environ. Sci. 9, 1439-1449 (2016).

47. Li, F. et al. Ambipolar solution-processed hybrid perovskite phototransistors. Nat. Commun. 6, 8238 (2015).

48. Bube, R. H. Photoelectronic Properties of Semiconductors (Cambridge University Press, Cambridge, UK, 1992).

49. Herz, L. M. Charge-carrier dynamics in organic-inorganic metal halide perovskites. Annu. Rev. Phys. Chem. 67, 65-89 (2016).

50. Zhao, T., Shi, W., Xi, J., Wang, D. \& Shuai, Z. Intrinsic and extrinsic charge transport in $\mathrm{CH}_{3} \mathrm{NH}_{3} \mathrm{PbI}_{3}$ perovskites predicted from first-principles. Sci. Rep. 6, 19968 (2016).

51. Rothmann, M. U. et al. Direct observation of intrinsic twin domains in tetragonal $\mathrm{CH}_{3} \mathrm{NH}_{3} \mathrm{PbI}_{3}$. Nat. Commun. 8, 14547 (2017).

52. Wang, G. et al. Wafer-scale growth of large arrays of perovskite microplate crystals for functional electronics and optoelectronics. Sci. Adv. 1, e1500613 (2015)

\section{Acknowledgements}

The work at UT-Austin led by X.L. and K.L. is supported by NSF EFMA-1542747. Z.C and D.W. also acknowledge the support from Welch Foundation Grant No. F-1814. The work at the National Renewable Energy Laboratory was supported by the U.S. Department of Energy under Contract No. DE-AC36-08-GO28308. K.Z., M.Y., and P.S. acknowledge the support by the hybrid perovskite solar cell program of the National Center for Photovoltaics funded by the U.S. Department of Energy, Office of Energy Efficiency and Renewable Energy, Solar Energy Technologies Office.

\section{Author contributions}

K.L., X.L., and K.Z. conceived the project. M.Y., P.S., and K.Z. prepared samples and performed optical measurements. Z.C., X.M., and J.S. conducted the MIM measurements. Z.C. and K.L. performed data analysis and drafted the manuscript. The manuscript was written through contributions of all authors. All authors have given approval to the final version of the manuscript.

\section{Additional information}

Supplementary Information accompanies this paper at https://doi.org/10.1038/s41467017-02331-4.

Competing interests: K.L. holds a patent on the MIM technology, which is licensed to PrimeNano Inc. for commercial instrument. The terms of this arrangement have been reviewed and approved by the University of Texas at Austin in accordance with its policy on objectivity in research. The remaining authors declare no competing financial interests.

Reprints and permission information is available online at http://npg.nature.com/ reprintsandpermissions/

Publisher's note: Springer Nature remains neutral with regard to jurisdictional claims in published maps and institutional affiliations.

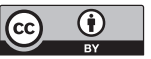

Open Access This article is licensed under a Creative Commons Attribution 4.0 International License, which permits use, sharing, adaptation, distribution and reproduction in any medium or format, as long as you give appropriate credit to the original author(s) and the source, provide a link to the Creative Commons license, and indicate if changes were made. The images or other third party material in this article are included in the article's Creative Commons license, unless indicated otherwise in a credit line to the material. If material is not included in the article's Creative Commons license and your intended use is not permitted by statutory regulation or exceeds the permitted use, you will need to obtain permission directly from the copyright holder. To view a copy of this license, visit http://creativecommons.org/ licenses/by/4.0/

(C) The Author(s) 2017 